\title{
Asymmetric Information and Monetary Policy in Common Currency Areas
}

\author{
Laura Bottazzi ${ }^{1} \quad$ Paolo Manasse ${ }^{2}$
}

June 2002

${ }^{1}$ Università Bocconi, IGIER and CEPR. Address: IGIER, Via Salasco 5, 20136 Milano, Italy. Email:laura.bottazzi@uni-bocconi.it

${ }^{2}$ Corresponding Author.Università di Bologna and IGIER. Address: Dept. of Economics, Strada Maggiore, 45, 40100 Bologna (Italy), E-mail:manasse@spbo.unibo.it 


\begin{abstract}
In a Common Currency Area (CCA) the Common Central Bank sets a uniform rate of inflation across countries, taking into account the area's economic conditions. Suppose that countries in recession favor a more expansionary policy than countries in expansion, a conflict of interest between members arises when national business cycles are not fully syncronized. If governments of member countries have an informational advantage over the state of their domestic economy, such conflict may create an adverse selection problem: national authorities overemphasize their shocks, in order to shape the common policy towards their needs. This creates an inefficiency over and above the one-policy-fits-all cost discussed in the optimal currency area literature. In order to minimize this extra-burden of asymmetric information, monetary policy must over-react to large symmetric shocks and under-react to small asymmetric ones. The result is sub-optimal volatility of inflation.
\end{abstract}




\section{Introduction}

In a common currency area (CCA) national governments delegate monetary policy decisions to a supra-national authority, the Common Central Bank (CCB). The CCB sets a uniform rate of inflation across countries, taking into account the area's economic conditions. Suppose that countries in recession favor a more expansionary policy than countries in expansion ${ }^{1}$. Then if national business cycles are not fully synchronized, a conflict of interest between members arises. When governments of member countries have an informational advantage over the state of their domestic economy, such conflict may create an adverse selection problem: national authorities overemphasize their shocks in order to shape the common policy towards their needs. Ignoring the problem can be extremely costly, since the CCB ends up inappropriately implementing "stop and go" policies that add to inflation variability. This cost adds over the standard one-policy-fits-all inefficiency related to the loss of an independent instrument for stabilization, as discussed in the optimal currency area literature. The paper's main result is that the optimal monetary policy must over-react to large (symmetric) shocks and under-react to small (asymmetric) ones. In order to provide the incentives for truthful revelation, inflation/deflation is exacerbated in very bad/good states. This policy ("separating rule") results in sub-optimal volatility of inflation and welfare loss. Moreover, we study the case when the CCB decides to ignore some of the information reported by national authorities. In particular, when the Central Bank conditions her policy only to the "sign" of the reports (expansion/recession), the resulting policy rule (the "pooling rule") aggravates the problem of inertia: it under-reacts to the area economic conditions, and inflation variability is even more sub-optimal.

The paper's central idea is that there is important asymmetric information between policymakers in different countries about their macroeconomic conditions. This can involve either outright misrepresentation of statistics (for example a country might misreport its unemployment rate, see below) or misinterpretation of statistics (for example national poli-

\footnotetext{
${ }^{1}$ This may be due to the existence of constraints to use domestic policy instruments for stabilization purposes. For example, balanced-budget rules, borrowing ceilings, limited inter-national redistribution schemes are frequent in currency unions between developed countries. Alternatively, this may be due to borrowing constraints and limited access to the international capital market, or insufficient national savings, as is often the case in developing economies.
} 
cymakers might be better placed than their foreign counterparts for judging the implications of labor market outcomes on the deviation of output from trend).

In his presidential address to the International Statistical Institute, Felligi (1989)[12] raises the issue quite clearly:

"..Statistical information is a product with peculiar attributes. One of them is that users are seldom in a position to check its quality directly. Yet data that are not trusted are clearly of little utility, whatever their intrinsic quality"

The case of the rate of unemployment in the UK is quite suggestive. In the 80's British labor economists have been bewildered by the ever-changing definition of unemployment. Paul Gregg [14](1994) recalls that

"..Charges ...against the count method of estimating unemployment have concerned allegations about politically inspired manipulation of the figures through numerous changes in coverage during the 1980s. The supporting evidence for these changes is that all but one of these changes have been unidirectional-downwards. Critics have expressed a view that calculating unemployment on the old (pre-1982) coverage would result in a considerably higher total figure.."2

Lack of confidence in the figures became so serious that a government Working Party was set up to analyze the question: according to the ensuing official report,

"The level of unemployment in a country is a key indicator of economic and social well-being. The UK figures published monthly are eagerly awaited and hotly debated. Recently, and especially

\footnotetext{
${ }^{2}$ According to Bartholomew at al.(1995) [2]

"...in the 1970s, the figure for unemployment broadly related to those receiving unemployment benefits, plus those who did not receive benefits but registered themselves regularly for possible work. Increasingly during the early 1980's the latter group was excluded from the count and the former group was tightened up. For examples, in 1981, some 195000 individuals were struck off the count by the removal of those in training or in temporary work; in 1982, a further 216000 individuals were struck off when benefit claimants only were included in the figures; in 1983, some 107000 men who were over 60 years of age, not working and not entitled to benefits or credits, were similarly struck off the count. An important consequence of these and other changes was the unease expressed by the general public about what the published figures actually meant and how the changes, which occurred both up or down, could be effectively assessed".
} 
during the early part of 1994, debate became intense and it was claimed, on behalf of the Opposition, that the figures were worthless. It was implied that they were manipulated by the government for its own political ends. This matter is of great concern for Society..."(Bartholomew, Moore, Smith, Allin (1995)) $[2]^{3}$

We think that these informational problems are potentially more serious at the international level, for arrangements such as currency unions and federal redistribution schemes. In this spirit Bordignon, Manasse and Tabellini (2001) [5] argue that

"...in the European Union or in countries such as Russia and China (Laffont (1995)([15])..., national or regional governments are the primary source of statistical information...while federal authorities are at a disadvantage in assessing the quality of this information" [5].

With the exception of Bottazzi and Manasse (1998) [6] the issue of asymmetric information in common currency areas has received no attention in the literature ${ }^{4}$. Dixit (2000, p.779) [10], however, mentions this among the "important extension(s) for future research" in the field of monetary policy in currency unions. Conversely, there exist a large public economics literature, particularly on fiscal federalism, that deals with problems of information. Recent examples include Bordignon, Manasse and Tabellini (2001) [5], who study inter-regional redistribution when tax bases are imperfectly observable by the federal government; Cremer at Al. (1995)[9], Bucovetsky et al.(1996)[4], Levaggi (1991)[16] deal with intergovernmental grants under asymmetric information over local preferences; Boadway et al.[3] (1995), Raff and Wilson (1995)[19] and Lockwood (1996)[18] study the issue of public goods provision when technology is imperfectly observable. Finally, Laffont[15](1995) studies fiscal arrangements in China.

The plan of the paper is the following. Section 2 presents the model. Section 3 considers the benchmark case of full-information, when each country maintains its own currency. Section

\footnotetext{
${ }^{3}$ Another example is the elimination of the interest rate on mortgages from the definition of the CPI in 1993 in Britain. This was done in order to prevent inflation form shooting up whenever interest rates were raised. Avinash Dixit jokingly tolds us about the "Dixit and Goodhart" definition of "core" inflation: the index covering all goods whose price have not increased (!). Other interesting stories on statistics can be read on the BBC site: http://www.stats.org/statswork/bbc-stats.htm

${ }^{4}$ With respect to our previous work, here we do not insist on microfoundations, and change the equilibrium concept from dominant to bayesian strategies.
} 
4 discusses a Common Currency Area (CCA) with full information over economic conditions in every member country. In Section 5 we extend the set-up to asymmetric information. First we show that the full information policy is not incentive compatible, and we discuss the potential costs of ignoring the problem of information. We then discuss two alternative policy rules the solve the problem. The first policy "separates" the types, while the second "pools" them. Section 6 briefly discusses some possible extensions of the model, and Section 7 summarizes the main results.

\section{The Model}

There are two endowment-economies, each populated by an identical representative agent. The agent's indirect utility function, $W$, depends on inflation $\pi$, and on an output shock, $e$, representing the deviation of output from trend. A negative realization means that the economy is in "recession", a positive one that the economy is in "expansion". The output shocks are independent across countries. We require the indirect utility function $W(\pi, e)$ to have some intuitive properties:

$$
W_{e}(\pi, e)>0, W_{\pi}(0, e)>0, W_{\pi \pi}(\pi, e)<0, W_{\pi e}(\pi, e)<0
$$

where $W_{x}($.$) denotes the partial derivative of W$ with respect to $x$. The first property captures the positive effects of output on consumption. The second and the third represent the idea that, at low level of inflation, $\pi$ is beneficial; above a certain threshold, however, the benefits peter out and the costs of inflation increase. Notice that, due to the last inequality, a country's most preferred rate of inflation falls when the state of the economy improves. In the appendix we present a dynamic general equilibrium model of the moneyinto-the utility-function type, where welfare in steady state exhibits such properties. For this model, adapted from Bottazzi and Manasse(2002)([7]), seigniorage is an efficient way of financing the provision of a public good, when inflation is low. When inflation is high, however, distortions in money demand and declining marginal utility of the public good set in, and reduce welfare. Moreover, the preferred rate of inflation declines with output for the following reason: high output yields high fiscal revenue and reduces the need of seigniorage revenue. For expositional purposes we assume that the indirect utility function takes the 
quadratic form

$$
W(\pi, e)=-\frac{1}{2} \pi^{2}-e \pi+f(e), f_{e}>0
$$

and assume that $e$ can take a finite number of realizations in the interval $[-s, s], s>0$ with probabilities $p(e), \sum_{e} p(e)=1$, expected value $E(e)=0$, and variance $\sigma_{e}^{2}$. The disturbances across countries, $e$ and $e^{\prime}$ ("the other's" state), are independent. It will be very convenient to consider the particular case where $e$ can take only four possible realizations, $[-s,-s / 2, s / 2, s]$, with equal probability $p(e)=1 / 4$. Under the assumption ${ }^{5}$ of discrete uniform distribution, the shocks have zero mean, and variance $\sigma_{e}^{2}=(5 / 8) s^{2}$.

\section{Monetary Independence}

Next we characterize the optimal policy when each country has monetary sovereignty. We show that the optimal rule is counter-cyclical. After the realization of the shock, the national central bank chooses inflation so as to

$$
\underset{\pi}{\operatorname{Max}} W(\pi, e)
$$

The optimal inflation rule is simply given by

$$
\pi(e)=-e
$$

The first-best rule is to run an expansionary policy in bad times and a contractionary policy in good times. We can calculate the expected utility in this benchmark case, as well as the first two moments of inflation ${ }^{6}$ :

$$
\begin{aligned}
& U(\pi(.))=\sum_{e} p(e) W(\pi(e), e)=\frac{\sigma_{e}^{2}}{2}=\frac{10}{32} s^{2}=0.312 s^{2} \\
& E(\pi(e))=\sum_{e} p(e) \pi(e)=0, \quad \sigma_{\pi}^{2}=\sigma_{e}^{2}=\frac{20}{32} s^{2}=0.625 s^{2}
\end{aligned}
$$

In the benchmark of monetary independence the average inflation rate is zero, and its variability exactly matches that of the "fundamentals".

\footnotetext{
${ }^{5}$ We carry on with the more general notation $p(e)$ since we discuss a more general case later on.

${ }^{6}$ We normalize the $f(e)$ function so that $\sum_{e} p(e) f(e)=0$
} 


\section{CCA under Full Information}

Next we describe the optimal policy in a CCA when the two states $e, e^{\prime}$ are fully observable. We show that the standard inefficiency of the Optimal Currency Area literature arises for the impossibility to tailor the common policy to individual needs. In a Common Currency Area, monetary policy is set by common central bank, the CCB, which chooses a common inflation rate, after observing the realizations of the shocks in the two countries. Thus the CCB solves

$$
\underset{\pi}{\operatorname{Max}}\left(W(\pi, e)+W\left(\pi, e^{\prime}\right)\right)
$$

The optimal policy rule is

$$
\pi^{E}\left(e, e^{\prime}\right)=-\frac{e+e^{\prime}}{2}
$$

What matters now is the aggregate (mean) state of the economy. This rule has some intuitive properties: i) it treats both types equally (it is "fair"), $\pi^{E}\left(e, e^{\prime}\right)=\pi^{E}\left(e^{\prime}, e\right)$; ii) it is symmetric, $\pi\left(e, e^{\prime}\right)=-\pi\left(-e,-e^{\prime}\right)$; iii) it is non-increasing in the shocks, $\pi_{e}^{E}\left(e, e^{\prime}\right) \leq 0$, so that inflation is not raised when more favorable shock are reported, and iv) it coincides with the first-best rule when shocks are identical, $\pi^{E}(e, e)=\pi(e)$. The last two properties show the nature of the conflict of interest between countries, when shocks differ: the country experiencing a recession ("boom") favors a looser (tighter) monetary policy than the one implemented by the CCB: $\pi\left(e^{\prime}\right)>\pi^{E}\left(e, e^{\prime}\right)>\pi(e)$ for $e>e^{\prime}$. Thus the rule gives rise to the standard inefficiency of currency unions, the one-policy-fits-all, whenever shocks are asymmetric. We can compare the two regimes by computing welfare:

$$
\begin{aligned}
& U\left(\pi^{E}(.)\right)=\sum_{e} \sum_{e^{\prime}} p(e) p\left(e^{\prime}\right) W\left(\pi^{E}\left(e, e^{\prime}\right), e\right)=\frac{5}{32} s^{2}=0.156 s^{2} \\
& E\left(\pi^{E}(.)\right)=\sum_{e} \sum_{e^{\prime}} p(e) p\left(e^{\prime}\right) \pi^{E}\left(e, e^{\prime}\right)=0, \quad \sigma_{\pi^{E}}^{2}=\sigma_{e}^{2} / 2=\frac{10}{32} s^{2}=0.312 s^{2}
\end{aligned}
$$

With respect to the regime of Monetary Independence, the CCA reduces welfare by $50 \%{ }^{7}$ $\left(U\left(\pi^{E}\right) / U(\pi)=1 / 2\right)$. The loss doesn't not stem from the average level of inflation, which

\footnotetext{
${ }^{7}$ This number must be treated with caution, since it relies on our normalization $\sum p(e) f(e)=0$. Had we normalized this expression to another constant, we would have obtained a different number.
} 
is the same in the two regimes, but from its inertia (i.e. sub-optimal inflation variability): since shocks are independent, the policy rule cannot match the variability of the state of each individual economy. This is the standard loss of an independent policy tool for stabilization.

\section{Asymmetric Information}

Suppose now that both governments have an informational advantage regarding the state of their own economy: the domestic government observes $e$ but not $e^{\prime}$, and viceversa the foreign one. The CCB cannot verify either one. In such circumstances, the policy rule of the CCB must be contingent on the states reported by the two governments, $\widetilde{e}, \widetilde{e}$. Each government may try to exploit its informational advantage in order to induce the CCB to choose a policy that better fits the state of its own economy. If the CCB ignores this incentive, it ends up choosing the "right" policy for the "wrong" state. Therefore, she must design a policy rule such that truthful revelation occurs. Next we show that, in order to "separate" the types and extract the correct information, the rule must over-react to large symmetric shocks and under-react to small asymmetric ones.

\subsection{The game}

Let's be more precise about the game being played. The timing is the following. In stage 1 the CCB designs a policy rule $\pi(\widetilde{e}, \widetilde{e})$ that depends on the reports. In stage 2 the shocks are realized, but only national governments observe national realizations. In stage 3 , given the policy rule, countries simultaneously choose a report in order to maximize their own expected utility, where the expectation is taken over the other country's report. Finally, the CCB implements the rule according to the reported states.

The optimal reporting strategy of "type" $e, R(e, \pi(\widetilde{e}, \widetilde{e}))$ consists in choosing the state to report so as to maximize his own expected utility, taking the expectations over the other country's report:

$$
R\left(e, \pi\left(\widetilde{e}, \widetilde{e}^{\prime}\right)\right)=\arg \max _{\widetilde{e}} E_{\widetilde{e}^{\prime}} W(\pi(\widetilde{e}, \widetilde{e}), e)
$$


An equilibrium with truthful revelation is defined as a set of reports $\widetilde{e}, \widetilde{e}$ and policy rule $\pi\left(\widetilde{e}, \widetilde{e}^{\prime}\right)$, such that i) given the policy rule, each country chooses an optimal report, $R($.$) ; ii)$ reports are truthful, $R\left(e, \pi\left(\widetilde{e}, \widetilde{e}^{\prime}\right)=e\right.$; and iii) the policy rule is optimal, in the sense that it maximizes expected welfare:

$$
\pi\left(\widetilde{e}, \widetilde{e}^{\prime}\right)=\arg \max _{\pi} U\left(\pi\left(\widetilde{e}, \widetilde{e}^{\prime}\right)\right)
$$

The policy can be viewed as a contract that the two parties (the governments) sign before the realization of the shocks. The contract describes the rate of inflation that the CCB must implement in all possible contingencies.

\subsubsection{Incentives to lie}

Here we show that the second-best rule $\pi^{E}().(7)$ does not induce truth-telling, i.e. it is not incentive-compatible. Intuitively, given this rule, each country is tempted to overemphasize its shock, so as to pull the rate of inflation closer to its most preferred rate $\pi(e)$. But then, if the governments systematically lie to the CCB, and CCB insists on applying $\pi^{E}$, the consequences are "disastrous". The CCB can do better, but at the cost of introducing some distortions in her policy rule.

To see this, first we need to calculate the optimal report when the rule $\pi^{E}($.$) is implemented$ With the aid of equation (7), write the country's indirect utility (2) as follows:

$$
W\left(\pi^{E}(\widetilde{e}, \widetilde{e}), e\right)=-\frac{1}{2}\left(-\frac{\widetilde{e}+\widetilde{e}^{\prime}}{2}\right)^{2}-e\left(-\frac{\widetilde{e}+\widetilde{e}^{\prime}}{2}\right)+f(e)
$$

Having observed the realization of his own state, type $e$ chooses his report by forming an expectation on $\widetilde{e}$. Expected utility of type $e$ is therefore

$$
E_{\widetilde{e}^{\prime}} W\left(\pi^{E}(\widetilde{e}, \widetilde{e}), e\right)=-\frac{1}{8}\left(\widetilde{e}^{2}+E_{\widetilde{e}^{\prime}}\left(\widetilde{e}^{2 \prime}\right)+2 \widetilde{e} E_{\widetilde{e}^{\prime}}(\widetilde{e})\right)+\frac{e \widetilde{e}}{2}+\frac{e E_{\widetilde{e}^{\prime}}\left(\widetilde{e}^{\prime}\right)}{2}+f(e)
$$

The optimal report $R$ is found by choosing the value of $\widetilde{e}$ that maximizes the above expres$\operatorname{sion}^{8}$. The first order condition for this problem is:

$$
R=2 e-E_{\widetilde{e}^{\prime}}(\widetilde{e})=2 e-E\left(R^{\prime}\right)
$$

\footnotetext{
${ }^{8}$ To deliver the intuition we look at the limit case when $e$ is a continuous variable.
} 
where to save on notation, we write $E\left(R^{\prime}\right)=E_{\widetilde{e}^{\prime}}\left(\widetilde{e}^{\prime}\right)$, and, for the other's expectation, $E^{\prime}(R)=$ $E_{\widetilde{e}}(\widetilde{e})$. From this expression it is easy to see that the policy rule $\pi^{E}$ does not induce truthtelling under rational expectations. The proof runs as follows. Each type would report the true state, $R=e$ only provided $E\left(R^{\prime}\right)=e$ holds. Since, by symmetry, the other player's optimal report satisfies $R^{\prime}=2 e^{\prime}-E^{\prime}(R)$, it follows that $R=2 e-E\left(2 e^{\prime}-E^{\prime}(R)\right)=2 e-2 E\left(e^{\prime}\right)+$ $R$, by the law of iterated expectations $\left(E\left(E^{\prime}(x)\right)=E(x)\right)$. Thus $E\left(e^{\prime}\right)=E\left(R^{\prime}\right)=e$ must hold for truth-telling to be the optimal strategy: in order to report the true state each type must expect the other's shock to be perfectly correlated with his own. But this expectation is not rational, given that the shocks are known to be independent.Q.E.D.

The same argument establishes that $E\left(R^{\prime}\right)=E\left(e^{\prime}\right)=0$, i.e. the optimal report has the same expected value of the true shock. But then, types $\pm s / 2$ will lie and, following (13) they will report, $\pm s$, respectively: when the policy $\pi^{E}$ is followed, only "extreme" shocks will be declared. Note that those who experience the extreme states for real are constrained to report the truth, since $e= \pm 2 s$ simply does not exist. Moreover, types $\pm s$ have no scope for lying: if they report an intermediate state, they end up pushing the CCB in the "wrong" direction". 9 Finally, observe that since only states $s$ and $-s$ will be reported, with equal probability, the expectation $E\left(R^{\prime}\right)=E\left(e^{\prime}\right)=0$ is indeed rational under the rule $\pi^{E}$. The intuition is straightforward. Type $-s / 2$ expects the other to report zero on average, so he knows that if he declares the truth and the CCB follows the rule $\pi^{E}\left(e, e^{\prime}\right)$, he will get, on average, $\pi^{E}=s / 4$. But this policy is "too tight" for him (he prefers $\pi(-s / 2)=s / 2>s / 4$ ). In order to induce the $\mathrm{CCB}$ to choose a looser policy, he declares that the economy is experiencing a very deep recession (and report $-s$ instead). By so doing (if the CCB believes him) he expects to get away with his first best, $\pi^{E}(-s, 0)=\pi(-s / 2)=s / 2$. The same argument holds for type $s / 2$, who will always overemphasize the positive state and report a "boom" $s$, in order to get a tigher policy. ${ }^{10}$

\footnotetext{
${ }^{9}$ For example, type $-s$ likes a very expansionary policy $\pi(-s)=s$, and, if he say the truth, he expects to get a rate of inflation $\pi^{E}=s / 2$ which is lower than desired. However, if he lies and reports, for example, $-s / 2$, he only harm himself, as he manages to induce an even less expansionary policy, $\pi^{E}=s / 4$.

${ }^{10}$ This argument exploits the fact that $E \pi^{E}()=.\pi^{E}(E()$.$) due to the linearity of \pi^{E}($.$) .$
} 


\subsubsection{The Dangers of "Closing the Eyes"}

The implication of this is striking: should the CCB "close her eyes" and adhere to the full information rule when governments cheat, she would end up alternating between extremely loose and extremely strict policies. She would alternate between $\pi^{E}(s, s)=-s$, $\pi^{E}(-s,-s)=s$, and $\pi^{E}(s,-s)=0$, the wrong policies most of the time. With governments cheating, excess volatility would result, and expected welfare $U^{C}\left(\pi^{E}\right)$ would only be

$$
\begin{aligned}
& U^{C}\left(\pi^{E}(.)\right)=\frac{4}{32} s^{2}<U\left(\pi^{E}(.)\right)=\frac{5}{32} s^{2} \\
& E^{C}\left(\pi^{E}(.)\right)=0, \quad \sigma_{\pi_{C}^{E}}^{2}=\frac{16}{32} s^{2}>\frac{10}{32} s^{2}=\sigma_{\pi^{E}}^{2}
\end{aligned}
$$

which corresponds to a $20 \%$ loss $\left(1-U^{C} / U^{E}=0.2\right)$ with respect to the full information outcome. Clearly, this stems from an excess inflation variability, which exceeds by $60 \%$ the full information benchmark $(16 / 10-1=0.6)$

\subsection{The "Separating" Inflation Rule}

The CCB can do better by designing a policy rule that is incentive compatible. We apply the revelation principle to our game, so that if an equilibrium of the game exists, it must also be a solution to the following problem: the CCB chooses a policy rule that maximizes expected welfare subject to the incentive compatibility constraints. We impose the reasonable restriction that the rule must satisfy the properties of "fairness", "symmetry" and that of being "non increasing in the shocks", i)-iii), discussed in Section 4. Hence we need only to consider the incentive compatibility constraint of types $s / 2$ (and $-s / 2$ ). Formally, the optimal rule solves Problem 1:

$$
\begin{aligned}
\max _{\pi\left(e, e^{\prime}\right)} U\left(\pi\left(e, e^{\prime}\right)\right) & =\sum_{e} \sum_{e^{\prime}} p(e) p\left(e^{\prime}\right) W\left(\pi\left(e, e^{\prime}\right), e\right) \\
s . t & \geq \sum_{e^{\prime}} p\left(e^{\prime}\right) W\left(\pi\left(s, e^{\prime}\right), s / 2\right) \\
\sum_{e^{\prime}} p\left(e^{\prime}\right) W\left(\pi\left(s / 2, e^{\prime}\right), s / 2\right) & \geq \sum_{e^{\prime}} p\left(e^{\prime}\right) W\left(\pi\left(-s, e^{\prime}\right),-s / 2\right)
\end{aligned}
$$


The incentive compatibility constraint (17) states that, under the rule, type $s / 2$ is at least as well off, in expected terms, by reporting $s / 2$ rather than $s$. A similar interpretation applies to equation (18) for type $-s / 2$.

The solution of this problem is simplified by noting that, because of the symmetry of the optimal rule, $\pi\left(e, e^{\prime}\right)=-\pi\left(-e,-e^{\prime}\right)$, either both constraints bind, or neither does. In the Appendix we prove the following proposition:

Proposition 1 Let $\pi^{S}\left(\widetilde{e}, \widetilde{e}^{\prime}\right)$ denote the inflation rule that solves Problem 1. When states of nature are equally likely, $p(e)=1 / 4$, all $e$, this policy satisfies the following properties: i) it over-reacts to large symmetric shocks, $\pi^{S}(-s,-s)>\pi^{E}(-s,-s)=s, \pi^{S}(s, s)<$ $\pi^{E}(s, s)=-s$; ii) it under-reacts to small asymmetric shocks, $\pi^{S}(s / 2,-s)<\pi^{E}(s / 2,-s)=$ $s / 4, \pi^{S}(-s / 2, s)>\pi^{E}(-s / 2, s)=-s / 4$; iii) the rule coincides with $\pi^{E}($.$) in the remaining$ cases $\pm(s / 2, s), \pm(s / 2, s / 2),(s / 2,-s / 2)$

\section{INSERT HERE FIGURE 1}

The proposition has an intuitive interpretation. The rule must make the intermediate types indifferent, in expected terms, between reporting the truth and lying. It does so in two ways (see Figure 1). First, in order to discourage extreme (false) reports, it makes inflation so high in symmetric bad states $(-s,-s)$, and so low in good ones $(s, s)$ that only countries who really experience these shocks may want to say so. This explains point $i$ ). At the same time, the policy encourages truthtelling by giving rents to the intermediate types in asymmetric states, e.g. $(s / 2,-s)$. Here the rule distorts the rate of inflation towards the center, i.e. towards the level preferred by the intermediate types. Notice that the alternative of punishing who reports $-s$ by further raising inflation would not work here, since then type $s / 2$ would have an even greater incentive to lie (and report $s$ ). This explains point $i i$ ). Finally, the policy does not need to distort the symmetric outcomes such as $\pm(s / 2, s), \pm(s / 2, s / 2)$ cf point $i i i)$, since the rule aims precisely at making types $\pm s / 2$ indifferent between telling the truth and reporting $\pm s$.

How effective is this rule for limiting the excess burden of asymmetric information? This 
can be discovered by calculating ${ }^{11}$

$$
\begin{aligned}
U\left(\pi^{S}\left(e, e^{\prime}\right)\right) & =\frac{4.961}{32} s^{2}=0.155 s^{2}<U\left(\pi^{E}(.)\right)=\frac{5}{32} s^{2} \\
E\left(\pi^{S}(.)\right) & =0, \quad \sigma_{\pi^{S}}^{2}=\frac{9.921}{32}=0.310 s^{2}<\frac{10}{32} s^{2}=\sigma_{\pi^{E}}^{2}
\end{aligned}
$$

Under the rule, welfare under asymmetric information falls below full information (but just $\left..06 \%\left(=1-U^{S} / U^{E}\right)\right)$. This happens because the rule exacerbates the "inertia" (suboptimal inflation variability) of the CCA.

\subsection{The "Pooling" Inflation Rule(s)}

There is an alternative way for CCB to eliminate the incentive to lie. It suffices to choose a rate of inflation that "pools" types $\pm s$ and $\pm s / 2$. The idea here is that the CCB only uses the information on the "sign" of the reported shocks, positive or negative, and completely disregards the information concerning the "size" of the shock. We show that this rule improves upon the cheating outcome, although it also leads to excessive inertia.

The optimal "pooling" rule solves Problem 2:

$$
\begin{aligned}
\max _{\pi\left(e, e^{\prime}\right)} U\left(\pi\left(e, e^{\prime}\right)\right)= & \sum_{e} \sum_{e^{\prime}} p(e) p\left(e^{\prime}\right) W\left(\pi\left(e, e^{\prime}\right), e\right) \\
& s . t \\
\pi(s / 2, s)= & \pi(s, s)=\pi(s / 2, s / 2)=\pi_{+}^{P} \\
\pi(s / 2,-s)= & \pi(s,-s)=\pi(s,-s / 2)=\pi(s / 2,-s / 2)=\pi_{+-}^{P}
\end{aligned}
$$

together with similar constraint for type $-s / 2^{12}$

The rule makes type $s / 2$ indifferent between reporting the true state or state $s$ simply by choosing the same level of inflation irrespective of the two reports. It is immediate to show the following:

\footnotetext{
${ }^{11} \mathrm{We}$ assume that indifference is enough to induce truth telling in the calculation below.

${ }^{12}$ Alternatively, we can simply impose the symmetry and "fairness" requirements that $\pi\left(e, e^{\prime}\right)=$ $-\pi\left(-e,-e^{\prime}\right)$, and $\pi\left(e, e^{\prime}\right)=\pi\left(e^{\prime}, e\right)$.
} 
Proposition 2 Let $\pi^{P}\left(\widetilde{e}, \widetilde{e}^{T}\right)$ denote the inflation rule that solves Problem 2. Under a discrete uniform distribution of shocks, $p(e)=1 / 4$ all $e$, this policy satisfies the following properties: $\pi_{+}^{P}=\pi^{E}(s / 2, s)=-(3 / 4) s ; \pi_{+-}^{P}=0$

The reason for this result is quite simple: the three possible outcomes in the two constraints are equally likely, so that the CCB chooses the inflation rate that is optimal in the intermediates states , $(s / 2, s),(-s / 2, s / 2)$. A different policy would strongly penalize intermediate types and further reduce welfare. It is possible to evaluate the implications of this rule by computing the expected utility $U\left(\pi^{P}\left(e, e^{\prime}\right)\right)$

$$
\begin{aligned}
U\left(\pi^{P}\left(e, e^{\prime}\right)\right) & =\frac{4.5}{32} s^{2}=0.141 s^{2}<U\left(\pi^{E}(.)\right)=\frac{5}{32} s^{2} \\
E\left(\pi^{P}(.)\right) & =0, \quad \sigma_{\pi^{P}}^{2}=\frac{9}{32} s^{2}=0.281 s^{2}<\frac{10}{32} s^{2}=\sigma_{\pi^{E}}^{2}
\end{aligned}
$$

The pooling rule is clearly preferable to the cheating outcome, (confront (23) and (14)). However, it is worse than the separating rule (confront (23) and (19)).since it aggravates the problem of inertia (i.e. sub-optimal inflation variability).

This is by no means the only pooling rule that eliminates the incentives to lie. One simple possibility for the CCB would be to ignore the informative content of each message and choose $\pi\left(e, e^{\prime}\right)=\pi$ for all $e, e^{\prime}$. The "optimal" inflation rate for this case would be $\pi^{P_{1}}=$ $\pi^{E}\left(E(e), E\left(e^{\prime}\right)\right)=0$, and lead to $U^{P_{1}}=\sigma_{\pi^{P_{1}}}^{2}=0$. By imposing unnecessary constraints, this policy rule is clearly inferior to the $\pi^{P}$ rule. ${ }^{13}$

\section{Extensions}

Next discuss some relevant extensions of the model. First we consider the case where small and large shocks occur with different probabilities. Second, we briefly discuss the case of many countries.

\footnotetext{
${ }^{13}$ Another pooling rule compatible with equilibrium is one that does not discriminate among small shocks of either sign, $\pi(-s / 2,-s / 2)=\pi(-s / 2, s / 2)=\pi(s / 2, s / 2)$.

Contrary to the $\pi^{P}$, however, such rule would not by itself eliminate the incentive to lie, so that incentive compatibility constraints similar to (17) should also be required. For this reason such rule will never be preferable to the separating rule.
} 


\subsection{Distribution of Shocks}

Suppose that "large" and "small" shocks occur with different probabilities. How does this affect the results? Two important things happen. First when the probability associated to either small or large shocks tends to zero, the full information solution applies: the CCB can safely ignore the reports concerning states that occur with zero probability.

Second, when large shocks becomes sufficiently likely, the welfare gain from adopting the separating rather than the pooling rule becomes smaller. Assume

$$
\begin{aligned}
p(s) & =p(-s)=p / 2 \\
p(s / 2) & =p(-s / 2)=(1-p) / 2
\end{aligned}
$$

with $0 \leq p \leq 1$. For example, if large shock are relatively rare events, $p<1 / 2$.

\subsubsection{Back to Full Information}

Consider the "extreme" cases when $p->0$ or $p->1$ : clearly the problem of asymmetry of information disappears. It is easy to show that when large shocks almost "never" occur, $p->0$, the CCB can safely disregard "large reports" and optimally chooses $\pi^{S}(s, s / 2)=$ $\pi^{E}(s / 2, s / 2), \pi^{S}(-s, s / 2)=\pi^{E}(-s / 2, s / 2)^{14}$. Similarly, if $p->1$, so that small shocks "never" occur, the CCB can safely disregard "small reports" and optimally chooses $\pi^{S}(s, s / 2)=$ $\pi^{E}(s, s)$.

\subsubsection{Separating vs Pooling Rules}

We are interested in comparing the welfare levels of the two policy rules, for different values of $p$. It is easy to show that the optimal "pooling" rule for the general case of distribution (24) is given by

$$
\pi_{+}^{P}=-\frac{s(1+p)}{2}, \pi_{+-}^{P}=0
$$

\footnotetext{
${ }^{14}$ In the (zero-probability) event of two large reports, for example $(s, s)$, any inflation rate (and among them $\left.\pi^{E}(s)\right)$ will do
} 
Clearly, the higher the probability of large shocks the higher (in absolute value) inflation under pooling. When $p=1 / 2$, the solution is $\pi_{+}^{P}=-(3 / 4) s$, as before (cf. Proposition 2). It is very difficult to find a closed form solution for $\pi^{S}$ for the case $p \in(0,1)$. However, we can resort to numerical solution (see Table 1 below) ${ }^{15}$ :

$\begin{array}{cccccccc}\mathbf{U} / \mathbf{p} & 0 & \mathbf{1} / \mathbf{8} & \mathbf{1} / \mathbf{4} & \mathbf{1 / 2} & \mathbf{3} / \mathbf{4} & \mathbf{7 / 8} & \mathbf{1} \\ \mathbf{U}\left(\boldsymbol{\pi}^{S}\right) & 0.062 s^{2} & 0.085 s^{2} & 0.108 s^{2} & 0.15502 s^{2} & 0.2022 s^{2} & 0.226 s^{2} & 0.25 s^{2} \\ \mathbf{U}\left(\boldsymbol{\pi}^{P}\right) & 0.062 s^{2} & 0.079 s^{2} & 0.098 s^{2} & 0.141 s^{2} & 0.192 s^{2} & 0.219 s^{2} & 0.25 s^{2} \\ \mathbf{U}\left(\boldsymbol{\pi}^{S}\right) / \mathbf{U}\left(\boldsymbol{\pi}^{P}\right)-\mathbf{1} & 0 & 7.6 \% & 10.8 \% & 10.2 \% & 5 \% & 3.2 \% & 0\end{array}$

Table 1: Numerical solution

In the extreme cases of only small $(p=0)$ or large $(p=1)$ shocks, the two policies are equivalent (we are effectively in the case of full information). If large and small shocks are equally likely, $p=1 / 2,(p(e)=1 / 4$, all $e)$ we are back to our discrete uniform distribution, with the $\boldsymbol{\pi}^{S}$ rule outperforming the $\boldsymbol{\pi}^{P}$ rule. Interestingly, the table shows that the relative gain from adopting the separating rather than the pooling rule first rises and then falls with the probability of large shock, $p$. The reason is as follows. The two rules give the full information outcome at the extremes, $p=0,1$ : however, welfare is increasing and concave in $p$ under separation, while it is increasing and convex under pooling, so that their ratio initially rises and then falls with $p$.

\subsection{Many Countries}

Having many countries in the Currency Area aggravates the distorsions of asymmetric information. Clearly, the standard inefficiency one-policy-fits all increases with the number of members, and so does the incentives to misreport information: hence larger distortions in the policy rule are required to prevent mimicking. Consider the separating rule first. In order

\footnotetext{
${ }^{15}$ In theAppendix we show that part i) of Proposition 1 goes through in non uniform case $p(e)$, i.e.the rule must over-react to large asymmetric shock. Similarly, the result that symmetric reports $(\widetilde{e},-\widetilde{e})$ are not distorted still applies. Finally, a sufficient condition for the rule to under-react to small asymmetric shocks (cf part ii of the proposition), is that the probability of large shocks $p \geq 1 / 2$. For the remaining cases (cf part ii of Proposition 1) the optimal policy distorts inflation towards the extremes or the middle depending on whether $p>$ or $<1 / 2$, respectively.
} 
to sketch the argument, let the CCA be formed by $j=1,2, \ldots N \geq 2$ identical countries, experiencing independent, discrete-uniformly distributed shocks $e_{j}$. The full information rule now is

$$
\pi^{E}(\mathbf{e}) \equiv \pi^{E}\left(e_{1, \ldots} e_{N}\right)=-\frac{1}{N} \sum_{j=1}^{N} e_{j}
$$

so that inflation has mean zero, and its variability $\sigma_{\pi}^{2}=\sigma_{e}^{2} / N$ tends to zero as $N$ goes to infinity. This implies, from the Central Limit Theorem, that the CCB will "always" choose an inflation rate equal to zero. It is easy to see that the incentive to overemphasize the shock rises with $N$. Proceeding as in Section $5.2^{16}$, the optimal report of country $i$ is now

$$
R_{i}=N e_{i}-E\left(\sum_{j \neq i} R_{j}\right)
$$

Compare (27) with (13): the larger the number of member countries, the larger the incentive to over-emphasize the shock. As $N$ grows sufficiently large, it must become increasingly costly to separate the types.

In the case of pooling, the three constraints in (21) must be replaced by $(N-1)+2=N+1$ constraints:

$$
\pi\left(s / 2, \underline{\mathbf{s}}_{1}\right)=\pi\left(s / 2, s / 2, \underline{\mathbf{s}}_{2}\right)=\ldots \pi\left(\underline{\mathbf{s}} / \mathbf{2}_{1}, s\right)=\pi(\underline{\mathbf{s}})=\pi(\underline{\mathbf{s} / \mathbf{2}})=\pi_{+}^{P}
$$

where $\underline{\mathbf{e}}$ is a realization of $N$ states equal to $e$, and $\underline{\mathbf{e}}_{n}$ is a realization where all but $n$ states (i.e. $N-n$ ) are equal to $e$. In summary, the distortions of both policy rules (of all rules) are likely to be fostered by the number of CCA members ${ }^{17}$.

${ }^{16}$ Under asymmetric information, the indirect utility of country $i$ under the rule (26) is

$$
\begin{aligned}
W\left(\pi^{E}, e_{i}\right)= & -\frac{1}{2 N^{2}}\left(\widetilde{e}_{i}^{2}+\left(\sum_{j \neq i} \widetilde{e}_{j}\right)^{2}+2 \widetilde{e}_{i} \sum_{j \neq i} \widetilde{e}_{j}\right) \\
& +\frac{e_{i} \widetilde{e}_{i}}{N}+\frac{e_{i} \sum_{j \neq i} \widetilde{e}_{j}}{N}
\end{aligned}
$$

Taking the expectation and solving for the optimal report, as in Sect.5.2, yields the expression in the text.

${ }^{17}$ Since a closed form solution for the separating rule becomes intractable, we were unable to compare the two rules for different values of $N$. 


\section{Discussion}

When members of a currency union experience idiosyncratic shocks, a conflict of interest over the stance of monetary policy arises. If governments have an informational advantage over the state of their domestic economy, this lead to a problem of adverse selection. National authorities overemphasize their shocks in order to shape the common policy towards their needs. Ignoring the problem can be extremely costly, since the monetary authority ends up inappropriately implementing "stop and go" policies that add to inflation variability and are detrimental to welfare. The paper shows that monetary policy must over-react to large symmetric shocks and under-react to small asymmetric ones. In order to provide the incentives for truthful revelation, monetary policy become unduly expansive in bad states and unduly restrictive in good ones, while it reacts too little to economic conditions in intermediate states. Overall, this aggravates the problem of sub-optimal volatility of inflation of CCA and causes a welfare loss. Alternatively, the Central Bank may decide to disregard some of the information reported by national authorities. In particular, when the Central Bank conditions her policy only to the "sign" of the reports (expansion/recession), the resulting policy rule is even more plagued by inertia: monetary policy under-reacts to the area economic conditions, and inflation variability is sub-optimal.

How robust are these conclusions? The model is clearly very stylized, yet we think that the conclusions are quite general: for example, the model can be given microfoundations (see the appendix). An interesting question for future research is whether intertemporal considerations may help reducing the distortions required for incentive compatibility. Along the lines of Atkenson and Lucas ([1]), one may conjecture that the CCB may induce truthtelling by conditioning her policy not only on current reports, but also on past ones, so that, for example, high inflation in a bad state today may come at the expenses of a lower inflation in a bad state tomorrow. The resulting dynamics of inflation over time are a topic worth investigating. 


\section{References}

[1] Atkenson, Andrew and Robert Lucas (1992), "On Efficient Distribution with Private Information", Review of Economic Studies, vol. 59, pp. 427-53.

[2] Bartholomew, David, Peter Moore, Fred Smith and Paul Allin, "The Measurement of Unemployment", (1995), Journal of Royal Statistical Society, Series A, n.158, part 3, pp.363-417

[3] Boadway R., Horiba I. and Raghbendra J.(1995), "The design of conditional grants as a principal agent problem", paper presented at the IIPF meeting, Lisboa, August 1995

[4] Bucovetsky S., Marchand M. and Pestieu(1996), "Tax competition and revelation of preferences for expenditures" (mimeo)

[5] Bordignon, M., P. Manasse and G.Tabellini (2001),"Optimal Regional Redistribution under Asymmetric Information", American Economic Review, Vol. 91 n.3

[6] Bottazzi , L. and P. Manasse, (1998)"Workers' versus Bankers' Europe (I): Asymmetric Information in EMU", IGIER Working Paper.

[7] Bottazzi , L. and P. Manasse, (2002)"Credibility and Seigniorage in A Common Currency Area", Journal of Money, Credit and Banking (forthcoming)

[8] Calvo, G.and P.E.Guidotti,(1993) "On the Flexibility of Monetary Policy: The Case of the Optimal Inflation Tax", The Review of Economic Studies, issue 3 , July, p.667-687

[9] Cremer H, Marchand M. and Pestieu(1994), "International redistribution through tax surcharge", Core Discussion Papers 9469

[10] Dixit,A. (2000) "A Repeated Game Model of Monetary Union", The Economic Journal,vol.110 $\mathrm{n}^{\circ} 466$

[11] Feenstra,Robert (1986)," Functional Equivalence between Liquidity Costs and the Utility of Money" Journal of Monetary Economics

[12] I.P. Felligi (1989), "Challenges to statistics and statisticians", Presidential Address to the International Statistical Institute 
[13] Fudenberg D. and J. Tirole (1991) Game Theory, Cambridge,MA:MIT Press

[14] Gregg, P.A. (1994) "Out for the count again!: A social scientists analysis of the Unemployment statistics in the U.K.", Journal of the Royal Statistical Society, Series A, Vol.157 No.2

[15] Laffont, J.J.,(1995), "Incentives in China's federal tax system" (mimeo) Essex University

[16] Levaggi, R,(1991)iscal Federalism and Grants in Aid, Aldershot:Averbury

[17] Laffont J.J. and J. Tirole(1996), "A Theory of Incentive in Procurement and Regulation" MIT Press

[18] Lockwood, B., (1996), "Inter-regional insurance with asymmetric information (mimeo)

[19] Raff, H. and Wilson, J.D., (1995), "Income redistribution with well informed local governments", (mimeo) Indiana University 


\section{Appendix}

\subsection{Microfoundations}

Here we provide a theoretical underpinning for the choice of our welfare function. Consider the following model. The world consists of two countries. Each country (domestic and foreign) is populated by a representative infinitely lived agent. We abstract from labor mobility. Both economies are endowed with one homogeneous good, $e_{t}$, which is defined in terms of random (i.i.d.). deviations from the trend $\bar{e}=0$ Each period, consumers face a given sequence of prices, tax rates and public spending, $\left\{p_{t}, g_{t}, \tau_{t}\right\}$, observe the realization of their endowment, and decide how much to consume, $c_{t}$, and save out of their disposable income. The endowment is taxed at a proportional rate, $\tau_{t}$. Households save by carrying nominal money balances, $M_{t+1}$ into next period. There is neither capital nor bonds in the economy. Preferences are described by an additively separable utility function, where separability is assumed both with respect to time and with respect to the arguments. Consumers choose the sequence $\left\{c_{t}, M_{t+1}\right\}_{0}^{\infty}$ so as to maximize the present discounted value of their utility stream ${ }^{18}$ :

$$
U=\sum_{t=0}^{\infty} \beta^{t} \mathcal{U}\left(c_{t}, \frac{M_{t+1}}{p_{t}}, g_{t}\right)=\sum_{t=0}^{\infty} \beta^{t}\left(u\left(c_{t}\right)+V\left(\frac{M_{t+1}}{p_{t}}\right)+H\left(g_{t}\right)\right)
$$

where $0<\beta<1$ denotes the rate of time preference, $\frac{M_{t+1}}{p_{t}}$ is the stock of domestic money balances at the beginning of period $t+1$, expressed in units of time $t$ goods, $V$ and $H$ are quasiconcave functions. Great simplification is obtained by assuming that utility is linear in consumption, $u\left(c_{t}\right)=c_{t}$ (cf. Calvo and Guidotti (1993)[8]). The consumer's budget constraint under Monetary Independence $(\mathrm{MI})$ is

$$
c_{t}+\frac{M_{t+1}}{p_{t}}=\left(1-\tau_{t}\right) e_{t}+\frac{M_{t}}{p_{t}}
$$

Similarly, in a CCA, the constraint reads

$$
c_{t}+\sigma \frac{\widetilde{M}_{t+1}}{p_{t}}=\left(1-\tau_{t}\right) e_{t}+\sigma \frac{\widetilde{M_{t}}}{p_{t}}
$$

\footnotetext{
${ }^{18}$ Since we need to justify why individuals hold real money balances, we need a model of intertemporal choice. An alternative approach would be to assume that money economizes on transaction costs. In this case real balances enter the budget constraint rather than the utility function. Under certain regularity conditions the two approaches are equivalent. See Feenstra (1986).
} 
where $\widetilde{M}_{t}=M_{t}+M_{t}^{*}$ represents the common currency, which is held by both domestic and foreign households $^{19}$, and $\sigma=1-\sigma^{*}$ represent the share of the common currency that is held by domestic consumers. For simplicity we assume that $e_{t}$, is a zero mean i.i.d. disturbance. Depending on the exchange rate regime, the national Central Bank (or the CCB) chooses the sequence of nominal balances, $\left\{M_{t}\right\}_{1}^{\infty}$ (or $\left.\left\{\widetilde{M}_{t}\right\}_{1}^{\infty}\right)$ given an initial value $M_{0}\left(\widetilde{M}_{0}\right)$.

\subsubsection{Government budget constraint}

National governments raise revenue by taxing the endowment at proportional rate $\tau_{t}$ and by seigniorage. For the sake of simplicity we do not consider the choice of $\tau_{t}$ which is assumed mandatory fixed and constant over time. Again, the budget constraint differs between monetary regimes. Under MI we have

$$
g_{t}=\tau e_{t}+\frac{M_{t+1}-M_{t}}{p_{t}}
$$

In turn, in a CCA, the constraint reads:

$$
g_{t}=\tau e_{t}+\sigma\left(\frac{\widetilde{M}_{t+1}-\widetilde{M}_{t}}{p_{t}}\right)
$$

By substituting the government constraint into the consumer constraint for MI and CCA respectively, one finds the resource constraint $c_{t}+g_{t}=e_{t}$

\subsubsection{Solution}

Solving the private sector maximization problem for the case of MI (29) subject to the constraint (30), and re-arranging the first order conditions for $c_{t}$ and $M_{t+1}$, recalling linearity, yields

$$
1=V^{\prime}\left(\frac{M_{t+1}}{p_{t}}\right)+\beta \frac{p_{t}}{p_{t+1}}
$$

This is a standard arbitrage condition that assures that no gains can be made by re-allocating consumption over time. Finally, we place the following restriction on the choice of the optimal

\footnotetext{
${ }^{19}$ Here we are assuming that the sequence of prices in the two countries is the same when they share the same currency.
} 
monetary policy. We assume that the Central Bank chooses the rate of money growth in order to achieve a rate of inflation that is a time-invariant function of the disturbance(s), so that $\widetilde{\pi}_{t}$ $=\frac{p_{t+1}-p_{t}}{p_{t}}=\tilde{\pi}\left(e_{t}\right)$. It is easy to see that this restriction implies that the rate of money growth, $\mu_{t}=\frac{M_{t+1}-M_{t}}{M_{t}}$ is also a time-invariant function of the disturbance(s) and equals $\tilde{\pi}(.)^{20}$. Thus we can write the demand for money and consumption in MI as follows:

$$
\begin{gathered}
\frac{M_{t+1}}{p_{t}}=m\left(\pi\left(e_{t}\right)\right), \text { all } t, \quad M_{\pi}<0 \\
c_{t}=(1-\tau) e_{t}-\pi\left(e_{t}\right) m\left(\pi\left(e_{t}\right)\right)
\end{gathered}
$$

where it is convenient to define $\pi()=.\frac{p_{t+1}-p_{t}}{p_{t+1}}=\frac{\widetilde{\pi}(.)}{1+\widetilde{\pi}(.)} 21$. The same conditions apply in a CCA, with $\sigma \widetilde{M}$ replacing $M$, and assuming that policy rule is a time-invariant function of both disturbances, $e, e^{*}$.These expressions immediately yield the indirect utility function $W$ in MI

$$
(1-\beta) W(\pi(.), e)=(1-\tau) e-\pi(.) m(\pi(.))+V(m(\pi(.))+H[\tau e+\pi(.) m(\pi(.))]
$$

and similarly for the CCA. The welfare function shows the nature of the optimal seigniorage problem of this economy. The inflation tax finances the provision of public goods, which are valued by consumers. Inflation, however, reduces real money holdings and induces distortions in the demand for money. It is immediate to show that this welfare function, under intuitive conditions, satisfies the assumptions in equation (1)

\subsection{Proof of Proposition 1}

Setting $p(e)=1 / 4$ in Problem 1 (15) gives the following first order conditions:

\footnotetext{
${ }^{20}$ From the first-order condition for real balances one can see that a constant rate of inflation implies constant money demand $\frac{M_{t+1}}{p_{t}}$. But since $\frac{M_{t+1}}{p_{t}} \equiv \frac{M_{t+1}}{p_{t+1}} \frac{p_{t+1}}{p_{t}}$, constant inflation and money demand require that $\frac{M_{t+1}}{p_{t+1}}$ is also constant. Thus in equilibrium the rate of money growth must equal the inflation rate.

${ }^{21}$ With a slight abuse of terminology, from now on we will call $\pi$, rather than $\tilde{\pi}$, the inflation rate. Also, given the stationarity of the policy rule, although not of the inflation rate, we suppress the time notation when it is not necessary.
} 


$$
\begin{array}{rll}
\pi_{-s,-s} & : W_{\pi}(-s, \pi)=\lambda W_{\pi}(-s / 2, \pi) \\
\pi_{-s .,-s / 2}: & {\left[W_{\pi}(-s, \pi)+W_{\pi}(-s / 2, \pi)\right]=-\lambda\left[W_{\pi}(-s / 2, \pi)-W_{\pi}(-s / 2, \pi)\right]} \\
\pi_{-s, s}: & {\left[W_{\pi}(-s, \pi)+W_{\pi}(s, \pi)\right]=\lambda\left[W_{\pi}(s / 2, \pi)+W_{\pi}(-s / 2, \pi)\right]} \\
\pi_{-s / 2,-s / 2}: & W_{\pi}(-s / 2, \pi)(1+\lambda)=0 \\
\pi_{-s / 2, s / 2}: & {\left[W_{\pi}(-s / 2, \pi)+W_{\pi}(s / 2, \pi)\right](1+\lambda)=0} \\
\pi_{-s / 2, s}: & {\left[W_{\pi}(-s / 2, \pi)+W_{\pi}(s, \pi)\right]=\lambda\left[W_{\pi}(s / 2, \pi)-W_{\pi}(-s / 2, \pi)\right]}
\end{array}
$$

where we have exploited the fact that the multipliers of the two incentive compatibility constraints are equal by symmetry: $\lambda^{+}=\lambda^{-}=\lambda$

Since the r.h.s. of (38) is zero, $\pi(-s,-s / 2)$ is not distorted and coincides with $\pi^{E}(-s,-s / 2)$. Also, from (41) we see that $\pi(-s / 2, s / 2)=0=\pi^{E}(-s / 2, s / 2)$. But then the r.h.s. of (39) is also zero, implying $\pi(-s, s)=0=\pi^{E}(-s, s)$. Thus we are left with equations (37) and (42). These, and the incentive constraint (18) can be written for our quadratic specification as follows:

$$
\begin{aligned}
(\pi(-s,-s)-s)-\lambda(\pi(-s,-s)-s / 2) & =0 \\
4 \pi(-s / 2, s)+s-2 \lambda s & =0 \\
s^{2} / 8+s \pi(-s / 2, s)+\frac{\pi(-s,-s)^{2}}{2}-(s / 2) \pi(-s,-s) & =0
\end{aligned}
$$

The solution gives $\pi^{S}(-s,-s)=1.09 s>s, \pi^{S}(-s / 2, s)=-0.174 s>-s / 4 ; \lambda^{S}=0.152$.

\subsection{Distribution of Shocks}

Proposition 1 is modified as follows

Proposition 2 Let $\pi^{S}\left(\widetilde{e}, \widetilde{e}^{\prime}\right)$ denote the inflation rule that solves Problem 1. When the distribution of states of nature is given by $(24)$ and $0<p<1$, this policy satisfies the following properties: i) $\pi^{S}($.$) over-reacts to large symmetric shocks (in the sense of Proposition 1) ; ii) a suf-$ ficient (but not necessary) condition for $\pi^{S}(\widetilde{e}, \widetilde{e})$ to under-react to small asymmetric shocks (as in Proposition 1$)$ is $p \geq 1 / 2$; iii) the rule coincides with $\pi^{E}($.$) in the cases \pm(s / 2, s / 2),(s / 2,-s / 2)$; iv) for the cases $\pm(s, s / 2)$ there are multiple solutions. When $p<1 / 2$, the policy associated to 
a global maximum distorts inflation towards the extremes $\left(\pi^{S}(-s,-s / 2)>\pi^{E}(-s,-s / 2)=\right.$ $\left.3 / 4 s, \pi^{S}(s, s / 2)<\pi^{E}(s, s / 2)=-3 / 4 s\right)$; when $p>1 / 2$ the distortion is towards the center : $\pi^{S}(-s,-s / 2)<\pi^{E}(-s,-s / 2), \pi^{S}(s, s / 2)>\pi^{E}(s, s / 2)$;finally when $p=1 / 2$, the policy rule $\pi^{S}$ is not distorted, as in Proposition 1

Proof. When the distribution is given by (24), the first-order conditions become:

$$
\begin{aligned}
\pi_{-s,-s}: & p W_{\pi}(-s, \pi)=\lambda W_{\pi}(-s / 2, \pi) \\
\pi_{-s .,-s / 2}: & \frac{p(1-p)}{2}\left[W_{\pi}(-s, \pi)+W_{\pi}(-s / 2, \pi)\right]=\lambda \frac{(1-2 p)}{2}\left[W_{\pi}(-s / 2, \pi)\right] \\
\pi_{-s, s}: & p\left[W_{\pi}(-s, \pi)+W_{\pi}(s, \pi)\right]=\lambda\left[W_{\pi}(s / 2, \pi)+W_{\pi}(-s / 2, \pi)\right] \\
\pi_{-s / 2,-s / 2}: & \left(\frac{1-p}{2}\right)^{2} W_{\pi}(-s / 2, \pi)\left(2+\left(\frac{1-p}{2}\right) \lambda\right)=0 \\
\pi_{-s / 2, s / 2}: & {\left[W_{\pi}(-s / 2, \pi)+W_{\pi}(s / 2, \pi)\right]\left(\frac{1-p}{2}\right)(1-p+\lambda)=0 } \\
\pi_{-s / 2, s}: & \frac{(1-p) p}{2}\left[W_{\pi}(-s / 2, \pi)+W_{\pi}(s, \pi)\right]= \\
& \lambda\left[\left(\frac{1-p}{2}\right) W_{\pi}(s / 2, \pi)-\left(\frac{p}{2}\right) W_{\pi}(-s / 2, \pi)\right]
\end{aligned}
$$

Comparing (43) with (37) we can see that the condition for $\pi(-s,-s)$ is unaffected by the change in the distribution. A similar condition holds for $\pi(s, s)$, so that part i) of Proposition 2 holds.

Comparing (44) with (38) we can see that when $p=1 / 2, \pi(-s,-s / 2)$ is not distorted, while if $p>1 / 2$, the r.h.s. of (44) is positive, since $W_{\pi}(-s / 2, \pi)<0$,so that $\pi(-s,-s / 2)$ is below the full information value, see part iv) of Proposition 2

Finally, comparing (47) with (41) we see that $\pi(-s / 2, s / 2)$ is the same as in full information. This and equation (46) implies that also $\pi(-s, s)$ from (45) is the same as under full information, cf point iii)

The case for small asymmetric shocks, $(-s / 2, s)$ is slightly more complex, since there are multiple solutions. Computing these solutions numerically and taking the one that delivers global maximum gives a value of $\pi(-s / 2, s)<s / 2$ (which is reasonable since the $\pi(-s / 2)=s / 2$ ) When this inequality is satisfied, we see that the r.h.s. of (48) $\left(\frac{2 p-1}{2}\right) \pi-\frac{s}{4}$ is surely negative for $p \geq 1 / 2$. Thus , under this condition, the l.h.s. of (48) is also negative, implying that $\pi^{A}(-s / 2, s)>\pi^{E}(-s / 2, s)$, cf part ii) of Proposition 2 


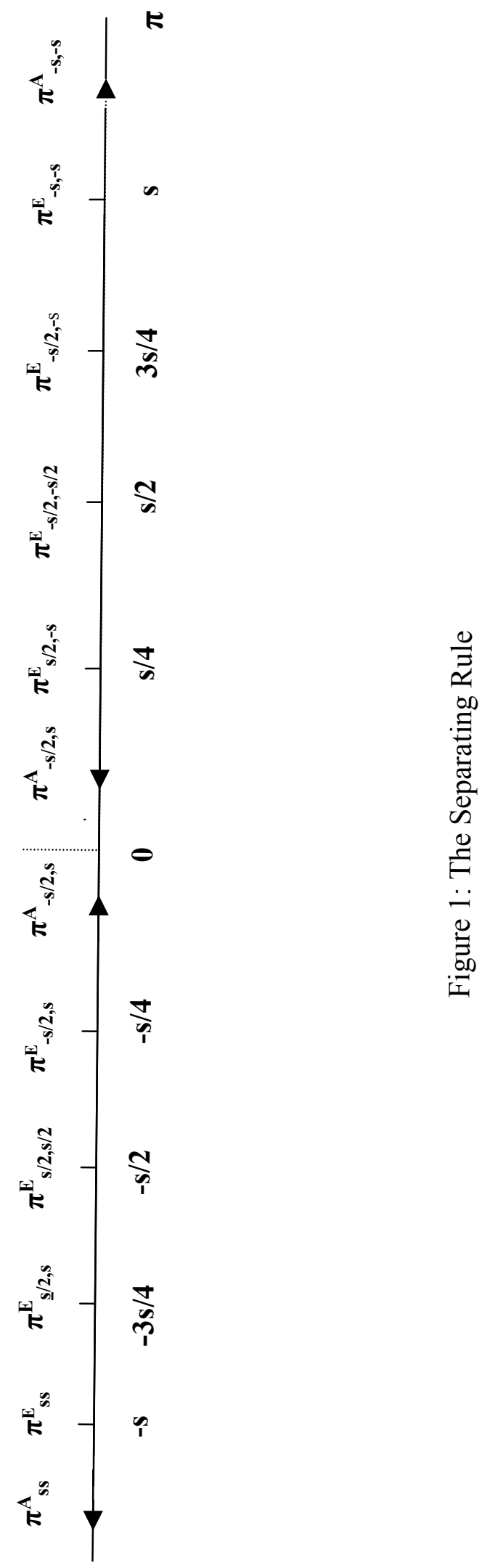

